\title{
Contributors to Volume 21
}

Numbers in parentheses indicate the pages on which the authors' contributions begin.

Frauke Baymann Aix Marseille University, CNRS, BIP (UMR 7281), 31 chemin Joseph-Aiguier, F-13402 Marseille, Cedex 20, France <baymann@imm.cnrs.fr> (135)

Stefanie Becker Geomicrobiology, Department of Geosciences, Universität Tübingen, Schnarrenbergstrasse 94-96, D-72074 Tübingen, Germany

<stefanie.becker@geo.uni-tuebingen.de> (185)

Elbert Branscomb Carl R. Woese Institute for Genomic Biology, and Department of Physics, University of Illinois, Urbana, IL 61801, USA $<$ brnscmb@illinois.edu $>$ (135)

Lena J. Daumann Department Chemie, Ludwigs-Maximilians-Universität, Butenandtstrasse 5, D-81377 München, Germany, <lena.daumann@lmu.de> (299)

Simon Duval Aix Marseille University, CNRS, BIP (UMR 7281), 31 chemin Joseph-Aiguier, F-13402 Marseille, Cedex 20, France<sduval@imm.cnrs.fr> (135)

Allison M. L. Enright Geomicrobiology, Department of Geosciences, Universität Tübingen, Schnarrenbergstrasse 94-96, D-72074 Tübingen, Germany <allison.m.enright@gmail.com> (185)

Christoph J. Fahrni School of Chemistry and Biochemistry, Petit Institute for Bioengineering and Bioscience, Georgia Institute of Technology, Atlanta, GA 30332, USA <fahrni@chemistry.gatech.edu> (81)

Shefali Harankhedkar School of Chemistry and Biochemistry, Petit Institute for Bioengineering and Bioscience, Georgia Institute of Technology, Atlanta, GA 30332, USA (81)

Andreas Kappler Geomicrobiology, Department of Geosciences, Universität Tübingen, Schnarrenbergstrasse 94-96, D-72074 Tübingen, Germany

<andreas.kappler@uni-tuebingen.de> (185)

Peter M. H. Kroneck Department of Biology, University of Konstanz, D-78457 Konstanz, Germany <peter.kroneck@uni-konstanz.de> (1) 
Ricardo O. Louro Instituto de Tecnologia Química e Biológica António Xavier, Universidade NOVA de Lisboa, Av. da República EAN, PT-2780-157 Oeiras, Portugal <louro@itqb.unl.pt> (229)

Shawn McGlynn Earth-Life Science Institute, Tokyo Institute of Technology, Ookayama, Tokyo, 152-8559, Japan <mcglynn@elsi.jp> (59)

Arielle Nabatilan School of Chemistry and Biochemistry, Petit Institute for Bioengineering and Bioscience, Georgia Institute of Technology, Atlanta, GA 30332, USA (81)

Wolfgang Nitschke Aix Marseille University, CNRS, BIP (UMR 7281), 31 chemin Joseph-Aiguier, F-13402 Marseille, Cedex 20, France $<$ nitschke@imm.cnrs.fr> (135)

Huub J. M. Op den Camp Department of Microbiology/72, Faculty of Science, Radboud University Nijmegen, Heyendaalseweg 135, P.O. Box 9010, NL-6525 AJ Nijmegen, The Netherlands <h.opdencamp@science.ru.nl> (299)

Catarina M. Paquete Instituto de Tecnologia Química e Biológica António Xavier, Universidade NOVA de Lisboa, Av. da República EAN, PT-2780-157 Oeiras, Portugal <cpaquete@itqb.unl.pt> (229)

Michael J. Russell NASA Astrobiology Institute, Ames Research Center, California, USA, and Dipartimento di Chimica, Università degli Studi di Torino, via P. Giuria 7, I-10125 Turin, Italy <michaeljrussel80@gmail.com> (135)

Bernhard Schink Department of Biology, University of Konstanz, D-78457 Konstanz, Germany < bernhard.schink@uni-konstanz.de> (33)

Barbara Schoepp-Cothenet Aix Marseille University, CNRS, BIP (UMR 7281), 31 chemin Joseph-Aiguier, F-13402 Marseille, Cedex 20, France $<$ schoepp@imm.cnrs.fr> (135)

Dirk Schüler Department of Microbiology, University of Bayreuth, D-95447 Bayreuth, Germany <dirk.schueler@uni-bayreuth.de> (159)

Martha E. Sosa Torres Departamento de Química Inorgánica y Nuclear, Facultad de Química, Universidad Nacional Autónoma de México, Ciudad Universitaria, México, D.F. 04510, México <mest@unam.mx> (1)

Inês B. Trindade Instituto de Tecnologia Química e Biológica António Xavier, Universidade NOVA de Lisboa, Av. da República EAN, PT-2780-157 Oeiras, Portugal <ines.trindade@itqb.unl.pt> (229)

René Uebe Department of Microbiology, University of Bayreuth, D-95447 Bayreuth, Germany <rene.uebe@uni-bayreuth.de> (159)

Anthony G. Wedd School of Chemistry and Bio21 Molecular Science and Biotechnology Institute, University of Melbourne, Parkville, Victoria 3010, Australia<agw@unimelb.edu.au> (271) 
Zhiguang Xiao Melbourne Dementia Research Centre, Florey Institute of Neuroscience and Mental Health, The University of Melbourne, Parkville, Victoria 3052, Australia, <zhiguang.xiao@florey.edu.au> (271)

Jiyao Yu School of Chemistry and Biochemistry, Petit Institute for Bioengineering and Bioscience, Georgia Institute of Technology, Atlanta, GA 30332, USA (81)

Kilian Zuchan Aix Marseille University, CNRS, BIP (UMR 7281), 31 chemin Joseph-Aiguier, F-13402 Marseille, Cedex 20, France <kzuchan@imm.cnrs.fr> (135) 
